\title{
An effective treatment option for pain caused by urolithiasis: A randomized-controlled trial of local active warming with heat-patch
}

huseyin mutlu ${ }^{1}$, Kamil Kokulu ${ }^{2}$, ekrem taha sert ${ }^{1}$, kasim $\operatorname{ertas}^{3}$, murat gul ${ }^{4}$, and mehmet akif diri ${ }^{1}$

${ }^{1}$ Aksaray Universitesi

${ }^{2}$ Aksaray University

${ }^{3}$ Van Yuzuncu Yil Universitesi

${ }^{4}$ Selçuk University

November 2, 2020

\begin{abstract}
Background: There is growing interest in physical medicine treatment options for renal colic. In this study, we aimed to determine whether or not heat-patch treatment with no drug was effective in relieving renal colic. Methods: For this purpose, patients who were diagnosed with renal colic in the emergency department were randomized to have either heat-patch or sham treatment. The Visual Analog Scale (VAS) scores of renal colic, body temperature (Btemp), and sub-patch skin temperature (Stemp) values were measured at $0,15,30,45$, and $60 \mathrm{~min}$. In addition, the salvage treatment needs of the groups were compared. Results: The average age of the study group was30.5 \pm 8.3 years and that of the sham group was $31.0 \pm 8.2$ years $(\mathrm{p}=0.75)$. According to the baseline VAS score of the patients, 15, 30, 45, and 60min VAS scores significantly decreased in the heatpatch group $(\mathrm{p}<0.001)$. The Btemp values did not differ significantly between the heat-patch and sham groups. In addition, no statistically significant difference was found between the two groups in terms of Stemp values at 0 and 15 min ( $\mathrm{p}=0.39 \mathrm{and}$ $\mathrm{p}=0.10$, respectively). However, there was a significant difference in the heat-patch group in terms of Stemp values at30, 45, and 60 min compared to the sham group $(\mathrm{p}<0.001)$. The salvage treatment rates for the heat-patch and sham groups were $11.5 \%$ and $31.4 \%$, respectively $(\mathrm{p}=0.01)$. Conclusion: As non-pharmaceutical treatment, the heat-patch has been shown to be a possible candidate for pain relief in patients with urolithiasis. Further research should concentrate on multicenter and large-scale randomized studies.
\end{abstract}

\section{An effective treatment option for pain caused by urolithiasis: A randomized-controlled trial of local active warming with heat-patch}

\section{ABSTRACT}

Background: There is growing interest in physical medicine treatment options for renal colic. In this study, we aimed to determine whether or not heat-patch treatment with no drug was effective in relieving renal colic.

Methods: For this purpose, patients who were diagnosed with renal colic in the emergency department were randomized to have either heat-patch or sham treatment. The Visual Analog Scale (VAS) scores of renal colic, body temperature (Btemp), and sub-patch skin temperature (Stemp) values were measured at 0,15,30,45, and $60 \mathrm{~min}$. In addition, the salvage treatment needs of the groups were compared.

Results: The average age of the study group was $30.5 \pm 8.3$ years and that of the sham group was $31.0 \pm$ 
8.2 years $(p=0.75)$. According to the baseline VAS score of the patients, 15, 30, 45, and 60min VAS scores significantly decreased in the heat-patch group $(p<0.001)$. The Btemp values did not differ significantly between the heat-patch and sham groups. In addition, no statistically significant difference was found between the two groups in terms of Stemp values at 0 and $15 \min (p=0.39$ and $p=0.10$, respectively). However, there was a significant difference in the heat-patch group in terms of Stemp values at30, 45, and 60 min compared to the sham group $(p<0.001)$. The salvage treatment rates for the heat-patch and sham groups were $11.5 \%$ and $31.4 \%$, respectively $(p=0.01)$.

Conclusion: As non-pharmaceutical treatment, the heat-patch has been shown to be a possible candidate for pain relief in patients with urolithiasis. Further research should concentrate on multicenter and large-scale randomized studies.

Keywords: Urolithiasis, Renal colic, Pain, Heat patch

\section{What is already known about this topic?}

The use of heat therapy in several diseases showed promising results. However, the use of heat therapy in the treatment of renal colic is scarce.

\section{What does this article add?}

The use of heat-band therapy for acute renal colic might be used in the emergency services as a first line treatment.

\section{Introduction}

For centuries, urolithiasis has been known for being one of the most painful diseases, and its prevalence is increasing (1). It is estimated that $8-15 \%$ of people around the world would face renal colic some time in their lives. In total, urolithiasis has been diagnosed in about $12 \%$ of the US population, and every year, there are approximately 2 million treatment visits at outpatient clinics for the disease. In the United States, renal colic accounts for $1 \%$ of all visits to the emergency department. However, for $50 \%$ of all patients with a history of renal calculi, this portion will rise to $50 \%$ after10 years $(1,3)$.

Renal colic occurs as a result of spasm because of the obstruction and distension caused by a calculus in the ureter (1). As recommended by the European Association of Urology, nonsteroidal anti-inflammatory drugs (NSAIDs) and opioids are first-line treatments for renal colic (4). However, they may cause renal injury by reducing renal blood circulation $(5,6)$. In addition, NSAIDs can cause gastrointestinal complications (such as ulcers and reflux) $(7,8)$. Their use is also limited in patients with hepatic failure and chronic obstructive pulmonary disease (5). Opioids - the other analgesic option-have a wide range of side effects, including nausea, vomiting, hypotension, sedation, dizziness, and even respiratory depression $(9,10)$.

In addition to these pharmaceutical agents, there are nonpharmaceutical alternative treatment methods that are used in relieving renal colic, including acupuncture, descending or ascending ladder, trigger point injection, Turkish bath, and local heating with a blanket $(11,13)$.

Heat-patch is an effective physical medicine treatment option designed to alleviate muscle pain by applying heat and is frequently used as physical therapy. The heat patch is quickly activated upon contact with air after being removed from its sheath. It contains no drugs, but iron powder, activated carbon, and water. Heat is generated when the iron particles inside the patch come in contact with air. The patch starts dissipating natural heat (long-term, 8h) several minutes after being targeted on the projective area of the pain. Patient satisfaction is high because it has an odorless and thin-dimension design.

Considering the success of heat-patch treatment among other nonpharmaceutical alternative treatment methods $(12-14,15)$. the aim of this study was to evaluate the efficacy of a heat-patch in relieving renal colic and to investigate its usability in daily treatment plans.

\section{Materials and methods}


This prospective study was designed as a randomized controlled, patient-blind, single-center clinical study held in Aksaray University Hospital between November 2018 and March 2019. The patients were provided detailed information about the study, and the informed consent form was signed. A total of 160 patients aged between 18 and 50 years and diagnosed with ureter stones by radiological imaging techniques (kidneybladder-ureter radiography, ultrasonography, or computed tomography) were subsequently registered for the study. Patients who had distal ureter stones (one-third of the last part of the ureter), were pregnant, had known previous allergy to skin patches, or had difficulty comprehending the Visual Analog Scale (VAS) were excluded from the study. In addition, patients who could not withstand pain and wanted analgesic agents were excluded. Out of 160, 42 patients failed to meet the inclusion criteria, and the remaining 118 patients who fulfilled the criteria for this study were offered participation by written information, and randomization was performed to receive either heat-patch or sham treatment (Fig.1). Randomization was conducted with a closed-envelope method. On the basis of the envelope selection, for patients who chose the letter $\mathrm{S}$, a heat patch $(9.5 \times 13.0 \mathrm{~cm})$ containing iron powder, activated carbon, and water (Voltapatch; GlaxoSmithKline Pharmaceuticals) was administered to the most painful region as indicated by the patients. For those who chose the letter C, a sham-patch-a cotton pad wrapped with gauze and designed to have the same size, weight, color, and temperature (room temperature) as that of a heat patch-was applied in a similar base. The patients in both groups did not receive any medical treatment outside the patch, but analgesic treatment ( $3 \mathrm{ml}$ of diclofenac sodium $75 \mathrm{mg}$ ) was initiated if the patient requested it during any period of the study. If the pain persisted, opioids (fentanyl $1.5 \mathrm{mcg} / \mathrm{kg}$, i.v.) were the second option.

All the patients enrolled in the study were evaluated with a detailed medical history and physical examination. Patches were attached to the locations with most pain. The VAS scores were evaluated for both groups at $0,15,30,45$, and 60 min after patch application. All the patients were monitored, and their vital findings were checked. Their body temperature (Btemp) values were measured with a tympanic thermometer (Braun ThermoScan IRT4520; Braun GmbH, Kronberg, Germany; range 34.0-42.2 ${ }^{\circ}$ ). The skin temperature (Stemp) values were measured using a thermometer (Nihon Kohden YSI-409A; Nihon Kohden, Tokyo, Japan) placed underneath the patch application site. At the end of this period, those who did not want to wait until the end and those who could not withstand pain and were given analgesic treatment ( $3 \mathrm{ml}$ of diclofenac sodium $75 \mathrm{mg}$, i.m.) were also excluded.

\subsection{Statistical analysis}

SPSS Statistics (v.15.0; SPSS Inc., Chicago, IL, USA) was used to perform statistical analyses. Numerical data were presented as means \pm standard deviation and medians (interquartile range). Frequency data were presented as $n$ and \%. The normal distribution of data was determined by the Kolmogorov-Smirnov test. The Mann-Whitney $U$ test and Student $t$ test were used to analyze comparisons between the two groups for numerical data. Homogeneity of the variance was tested using the Levene test. The mean and median differences were presented in a $95 \%$ confidence interval. The study was planned as a superiority trial, and all the analyses were performed according to the principle of intention to treat. All the hypotheses were constructed as two tailed, and an alpha critical value of 0.05 was accepted as significant. For this study, the sample size was calculated using the $G^{*}$ power software(v.3.1).In a similar study conducted earlier, it was found that the average VAS scores for pre- and posttreatment were 82.7 and 36.3, respectively, achieving the effect size of greater than one ${ }^{12}$. In this study, we aimed to reach at least 45 people in each group and 90 people in total in order to obtain a moderate effect size $(d=0.50)$ by predicting a smaller effect size.

\subsubsection{Ethical approval}

All the procedures performed in this study involving human participants were in accordance with the ethical standards of the Institutional and/or National Research Committee and with the Declaration of Helsinki,1964, and its later amendments or comparable ethical standards. Approval from the Institutional Review Board of Van Training and Research Hospital, Van, Turkey, was obtained prior to the execution of the study (2018/16). Written informed consent was obtained from all the patients. The study was reported in accordance with the Consolidated Standards of Reporting Trials statement, and the trial was registered at clinicaltrials.gov (NCT03790514). 


\section{Results}

A total of 103 patients (51 treated with a heat-patch and 52 sham) with renal colic were included in the study. The baseline characteristics between the two groups did not differ significantly (Table 1). The dropout rates for the two groups were $n=8(13.5 \%)$ and $n=7$ (11.9\%) patients, respectively (Fig.1).

On admission, both groups had similar VAS scores. The changes in VAS scores over time for the two groups are presented in Fig.2. In the heat-patch group, 15, 30, 45, and 60min VAS scores were significantly lower than those in the sham group. In addition, according to the baseline VAS score, the decrease in pain level in the heat-patch group at 15,30,45, and 60min was statistically significantly higher than that in the sham group. For both groups, 15, 30, 45, and 60 min VAS scores and changes in pain severity are provided in Table 2. Fourteen and six patients in the sham and heat-patch groups, respectively, needed pain salvage treatment $(p=0.01)$.

There was no statistically significant difference between the two groups in terms of $15,30,45$, and $60 \mathrm{~min}$ Btemp values $(p=0.17,=0.21,=23,=0.37$, and $=0.23$, respectively). In addition, there was no statistical difference found at 0 and $15 \mathrm{~min}$ Stemp values of the two groups ( $p=0.39$ and $p=0.10$, respectively). However, when Stemp was analyzed between the heat-patch and place groups at 30, 45, and 60 min, a statistically significant difference was found (Table 3 ).

\section{Discussion}

Nowadays, NSAIDs, paracetamol, and opioids are considered first-line treatments for renal colic; however, these drugs have many side effects. Besides, patients cannot directly access these medicines when the pain starts. Therefore, a simple and safe analgesic method during the prehospital phase could be useful for patients with renal colic. In this study, heat-patch treatment performed as a local warming method was shown to be superior to sham as an alternative treatment method for the treatment of renal colic. The changes in VAS scores were statistically significantly and clinically relevant.

Mechanosensitive receptors that are stimulated by mechanical induction, such as lithiasis, are the receptors that innervate the kidney and ureter mainly (16). However, unlike somatic pain, visceral pain is generally diffuse and poorly localized. It is also referred to as the body wall, where viscerosomatic convergence is the key in the central pain pathways. Visceral afferents are placed in only a few of the afferent inflows of the dorsal horn and viscerosomatic convergence in the dorsal horn, and supraspinal centers are very well documented. Therefore, visceral mechanosensitive receptors converge with heat afferents, raising from the body wall, and may change the central viscerosensory response in the dorsal column $(16,17)$. This hypothesis may explain the pain-relieving impact of body heating in this study. Another hypothesis that plays a role in reducing pain with the effect of heat may involve increased intravascular prostaglandins, bradykinin, and histamine secretion as a result of the increased blood flow to the region led by heat-patch treatment $(12-14,15)$.

In a preclinical trial, sympathetic blockading was found to be associated with attenuated visceral nociceptive responses. Pertovaara et al. demonstrated that following sympathectomy, visceral nociceptive responses decreased in rats (18). In addition, Kober et al. treated patients with renal colic by active warming of the lower back region and showed high sympathetic activity in patients with pain and urolithiasis (12). These findings highlight the role of the sympathetic nervous system in the perception of visceral pain.

Outside any situation of urolithiasis, local heat was recommended as a way of reducing the pain of trauma patients in the emergency department. In a study conducted by Bertalanffy et al. on patients with acute pelvic pain, where the other reasons for pelvic pain excluded gynecological causes, active heat treatment (electric blanket) was found to be a significantly effective method in reducing pelvic pain (19). Similarly, a study conducted by Nuhr et al. on patients with acute back pain, the pain was found to be reduced more in active heat treatment areas compared to passive heat treatment areas (15). In a randomized controlled study performed by Kober et al. on patients with renal colic, it was shown that VAS scores were statistically decreased in patients receiving active heat treatment compared to those receiving passive heat treatment ${ }^{12}$. Similar to these studies, in our study, we also showed that in the heat-patch group, Stemp values increased 
statistically, and the decrease in VAS scores was higher in the heat-patch group than in the sham group.

There are some inherent limitations in this study. The major limitation is the absence of comparison of heat-patch treatment and an analgesic agent. In addition, the fact that the patients received their actual treatment late could be considered a limitation; however, we were able to protect them from the possible side effects of the medications to be given. Another limitation that must be noted is that although we used patches that waited at room temperature for at least $24 \mathrm{~h}$ before the application in the sham group, we were aware that these patches would never reach the Stemp values of the study groups. However, no patient reported any heat-related complaints during the study. Last but not least, we did not include distal ureter stones, as the pain referred from these stones is mediated by the ilioinguinal and genitofemoral nerves, which radiate the pain to the groin, testicle, or labia majora.

\section{Conclusions}

Heat-patch treatment appears to be an ideal candidate to reduce pain in patients with urolithiasis, especially during the prehospital phase. As it does not contain any drug and has no side effects, healthcare professionals are not required for the application. We recommend that heat-patches be used at least as the primary treatment option for patients with urolithiasis before the actual treatment (if needed).

\section{REFERENCES}

1. Raheem OA, Khandwala YS, Sur RL, Ghani KR, Denstedt JD. Burden of Urolithiasis: Trends in Prevalence, Treatments, and Costs. Eur Urol Focus. 2017;3(1): 18-26.

2. Golzari SE, Soleimanpour H, Rahmani F, et al. Therapeutic approaches for renal colic in the emergency department: a review article.Anesth Pain Med. 2014;4(1): e16222.

3. Bultitude M. Urolithiasis around the world. BJU Int.2017;120(5): 601.

4. Türk C, Neisius A, Petř́k A, Seitz C, Thomas K, Skolarikos A. EAU Guidelines on Urolithiasis 2020. European Association of Urology Guidelines. 2020 Edition. Vol presented at the EAU Annual Congress Amsterdam 2020. Arnhem, The Netherlands: The European Association of Urology Guidelines Office; 2020.

5. McGettigan P, Henry D. Cardiovascular risk with non-steroidal anti-inflammatory drugs: systematic review of population-based controlled observational studies. PLoS Med. 2011;8(9): e1001098.

6. Lafrance JP, Miller DR. Selective and non-selective non-steroidal anti-inflammatory drugs and the risk of acute kidney injury.Pharmacoepidemiol Drug Saf. 2009;18(10): 923-931.

7. Davenport K, Timoney AG, Keeley FX. Conventional and alternative methods for providing analgesia in renal colic. BJU Int.2005;95(3): 297-300.

8. Bhala N, Emberson J, Merhi A, et al. Vascular and upper gastrointestinal effects of non-steroidal anti-inflammatory drugs: meta-analyses of individual participant data from randomised trials.Lancet. 2013;382(9894): 769-779.

9. Pathan SA, Mitra B, Romero L, Cameron PA. What is the best analgesic option for patients presenting with renal colic to the emergency department? Protocol for a systematic review and meta-analysis. $B M J$ Open. 2017;7(4): e015002.

10. Pathan SA, Mitra B, Straney LD, et al. Delivering safe and effective analgesia for management of renal colic in the emergency department: a double-blind, multigroup, randomised controlled trial. Lancet.2016;387(10032): 1999-2007.

11. Kaynar M, Koyuncu F, Buldu İ, et al. Comparison of the efficacy of diclofenac, acupuncture, and acetaminophen in the treatment of renal colic. Am J Emerg Med. 2015;33(6): 749-753.

12. Kober A, Dobrovits M, Djavan B, et al. Local active warming: an effective treatment for pain, anxiety and nausea caused by renal colic.J Urol. 2003;170(3): 741-744. 
13. Gul A, Gul M. Intracutaneous sterile water injection for pain relief during extracorporeal shock wave lithotripsy: comparison with diclofenac sodium. Urolithiasis. 2019.

14. Kober A, Scheck T, Fulesdi B, et al. Effectiveness of resistive heating compared with passive warming in treating hypothermia associated with minor trauma: a randomized trial. Mayo Clin Proc.2001;76(4): 369-375.

15. Nuhr M, Hoerauf K, Bertalanffy A, et al. Active warming during emergency transport relieves acute low back pain. Spine (Phila Pa 1976). 2004;29(14): 1499-1503.

16. Cervero F, Laird JM. Visceral pain. Lancet. 1999;353(9170): 2145-2148.

17. Al-Chaer ED, Traub RJ. Biological basis of visceral pain: recent developments. Pain. 2002;96(3): 221-225.

18. Pertovaara A, Kalmari J. Neuropathy reduces viscero-somatic inhibition via segmental mechanisms in rats. Neuroreport.2002;13(8): 1047-1050.

19. Bertalanffy P, Kober A, Andel H, Hahn R, Frickey N, Hoerauf K. Active warming as emergency interventional care for the treatment of pelvic pain. Bjog. 2006;113(9): 1031-1034.

\section{Legends to Figures and Tables}

Figure 1: Study flow diagram

Figure 2: Pain improvement at 15, 30, 45 and 60 minutes in study and sham group.

Table 1: Demographics and baseline characteristics of the study population.

Table 2: VAS scores at certain time points and change in pain intensity at 15, 30, 45 and 60 minutes for each study arm.

Table 3. Skin temperature alterations over time

CONSORT 2010 checklist of information to include when reporting a randomised trial*

\begin{tabular}{lll}
\hline Section/Topic & Item No & Checklist item \\
Title and abstract & Title and abstract & Title and abstract \\
& $1 \mathrm{a}$ & Identification as a randomised trial in the titl \\
& $1 \mathrm{~b}$ & Structured summary of trial design, methods, \\
Introduction & Introduction & Introduction \\
Background and objectives & $2 \mathrm{a}$ & Scientific background and explanation of ratio \\
& $2 \mathrm{~b}$ & Specific objectives or hypotheses \\
Methods & Methods & Methods \\
Trial design & $3 \mathrm{a}$ & Description of trial design (such as parallel, fa \\
& $3 \mathrm{~b}$ & Important changes to methods after trial com \\
Participants & $4 \mathrm{a}$ & Eligibility criteria for participants \\
& $4 \mathrm{~b}$ & Settings and locations where the data were co \\
Interventions & 5 & The interventions for each group with sufficier \\
Outcomes & $6 \mathrm{a}$ & Completely defined pre-specified primary and \\
& $6 \mathrm{~b}$ & Any changes to trial outcomes after the trial \\
Sample size & $7 \mathrm{a}$ & How sample size was determined \\
& $7 \mathrm{~b}$ & When applicable, explanation of any interim \\
Randomisation: & & \\
Sequence generation & $8 \mathrm{a}$ & Method used to generate the random allocatic \\
& $8 \mathrm{~b}$ & Type of randomisation; details of any restricti \\
Allocation concealment mechanism & 9 & Mechanism used to implement the random all \\
Implementation & 10 & Who generated the random allocation sequen
\end{tabular}


Blinding

Statistical methods

Results

Participant flow (a diagram is strongly recommended)

Recruitment

Baseline data

Numbers analysed

Outcomes and estimation

Ancillary analyses

Harms

Discussion

Limitations

Generalisability

Interpretation

Other information

Registration

Protocol

Funding
$11 \mathrm{a}$

$11 \mathrm{~b}$

$12 \mathrm{a}$

$12 \mathrm{~b}$

Results

$13 \mathrm{a}$

$13 \mathrm{~b}$

$14 \mathrm{a}$

$14 \mathrm{~b}$

15

16

$17 \mathrm{a}$

$17 \mathrm{~b}$

18

19

Discussion

20

21

22

Other information 23

24

25

If done, who was blinded after assignment to If relevant, description of the similarity of inte Statistical methods used to compare groups $\mathrm{fc}$ Methods for additional analyses, such as subg Results

For each group, the numbers of participants n For each group, losses and exclusions after rar Dates defining the periods of recruitment and Why the trial ended or was stopped

A table showing baseline demographic and cli For each group, number of participants (deno For each primary and secondary outcome, res For binary outcomes, presentation of both abs Results of any other analyses performed, inclu All important harms or unintended effects in Discussion

Trial limitations, addressing sources of potent Generalisability (external validity, applicabilit Interpretation consistent with results, balanci Other information

Registration number and name of trial registr Where the full trial protocol can be accessed, Sources of funding and other support (such as

*We strongly recommend reading this statement in conjunction with the CONSORT 2010 Explanation and Elaboration for important clarifications on all the items. If relevant, we also recommend reading CONSORT extensions for cluster randomised trials, non-inferiority and equivalence trials, non-pharmacological treatments, herbal interventions, and pragmatic trials. Additional extensions are forthcoming: for those and for up to date references relevant to this checklist, seewww.consort-statement.org.

\section{Hosted file}

Figure 1.pdf available at https://authorea.com/users/372350/articles/490429-an-effectivetreatment-option-for-pain-caused-by-urolithiasis-a-randomized-controlled-trial-of-localactive-warming-with-heat-patch 

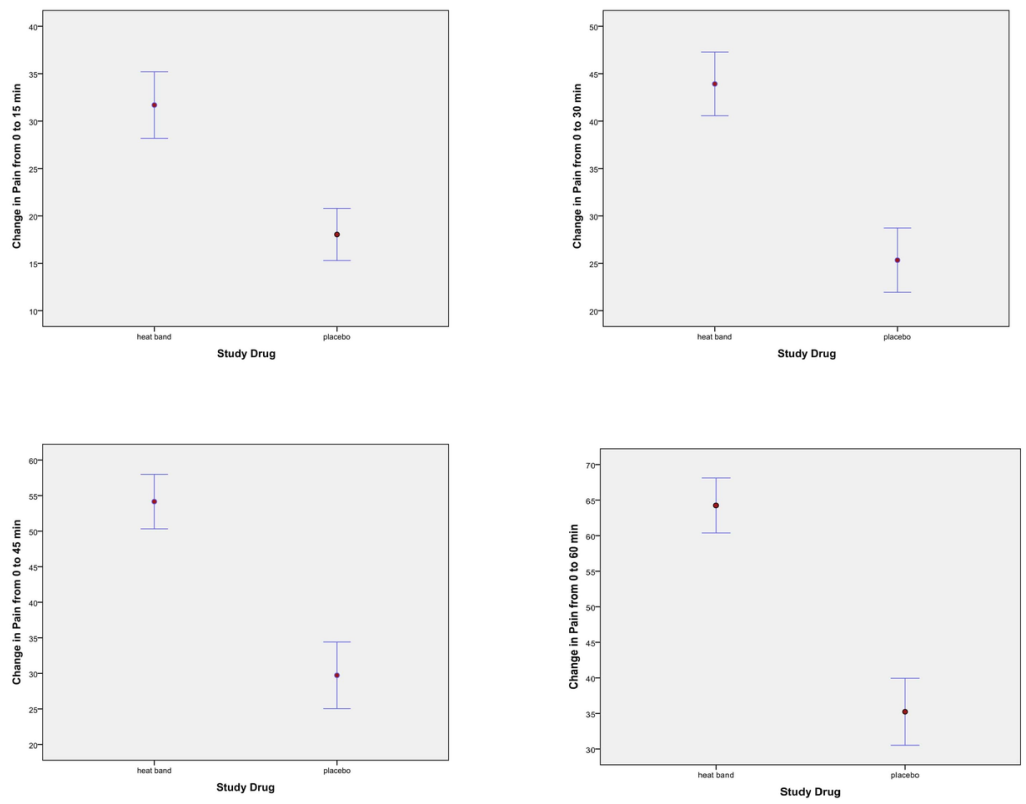

\section{Hosted file}

Table 1.pdf available at https://authorea.com/users/372350/articles/490429-an-effectivetreatment-option-for-pain-caused-by-urolithiasis-a-randomized-controlled-trial-of-localactive-warming-with-heat-patch

\section{Hosted file}

Table 2.pdf available at https://authorea.com/users/372350/articles/490429-an-effectivetreatment-option-for-pain-caused-by-urolithiasis-a-randomized-controlled-trial-of-localactive-warming-with-heat-patch

\section{Hosted file}

Table 3.pdf available at https://authorea.com/users/372350/articles/490429-an-effectivetreatment-option-for-pain-caused-by-urolithiasis-a-randomized-controlled-trial-of-localactive-warming-with-heat-patch 